\title{
The SLC16A11 risk haplotype is associated with decreased insulin action, higher transaminases and large-size adipocytes
}

\author{
Paloma Almeda-Valdes ${ }^{1,2}$, Donaji V Gómez Velasco', Olimpia Arellano Campos', \\ Omar Yaxmehen Bello-Chavolla1,3, Magdalena del Rocío Sevilla-González'1, Tannia Viveros Ruiz', \\ Alexandro J Martagón Rosado, Claudia J Bautista4, Liliana Muñoz Hernandez', Ivette Cruz-Bautista', \\ Hortensia Moreno-Macias 5,6, Alicia Huerta-Chagoya5, Karen Guadalupe Rodríguez-Álvarez', \\ Geoffrey A Walford7, Suzanne B R Jacobs7', Luz E Guillen Pineda², Ma Luisa Ordoñez-Sánchez ${ }^{5}$, \\ Ernesto Roldan-Valadez ${ }^{8,15}$, Joaquín Azpiroz9, Jannette Furuzawa-Carballeda ${ }^{10}$, Patricia Clark ${ }^{11}$, \\ Miguel F Herrera-Hernández ${ }^{12}$, Elena Zambrano4, Jose C Florez ${ }^{13,14}$, María Teresa Tusié Luna ${ }^{5}$ and \\ Carlos A Aguilar-Salinas ${ }^{1,2}$
}

${ }^{1}$ Unidad de Investigación en Enfermedades Metabólicas, Instituto Nacional de Ciencias Médicas y Nutrición Salvador Zubirán, Mexico City, Tecnológico de Monterrey, Escuela de Medicina y Ciencias de la Salud, Monterrey, N.L. México, ²Departamento de Endocrinología y Metabolismo, Instituto Nacional de Ciencias Médicas y Nutrición Salvador Zubirán, Mexico City, Mexico, ${ }^{3}$ MD/PhD (PECEM) Program, Facultad de Medicina, Universidad Nacional Autónoma de México, Mexico City, Mexico, ${ }^{4}$ Departamento de Biología de la Reproducción, Instituto Nacional de Ciencias Médicas y Nutrición Salvador Zubirán, Mexico City, Mexico, ${ }^{5}$ Unidad de Biología Molecular y Medicina Genómica, Instituto Nacional de Ciencias Médicas y Nutrición Salvador Zubirán/Instituto de Investigaciones Biomédicas UNAM, Mexico City, Mexico, ${ }^{6}$ División de Ciencias Sociales y Humanidades, Universidad Autónoma Metropolitana, Mexico City, Mexico, ${ }^{7}$ Programs in Metabolism and Medical \& Population Genetics, Broad Institute of Harvard and MIT, Cambridge, Massachusetts, USA, ${ }^{8}$ Directorate of Research, Hospital General de Mexico "Dr Eduardo Liceaga", Dr Balmis 148, Col. Doctores, Del. Cuauhtemoc, 06726 Mexico City, Mexico, ${ }^{9} \mathrm{Centro}$ Nacional de Investigación en Imagenología e Instrumentación Médica, Universidad Autónoma Metropolitana, Mexico City, Mexico, ${ }^{10}$ Departamento de Inmunología y Reumatología, Instituto Nacional de Ciencias Médicas y Nutrición Salvador Zubirán, Mexico City, Mexico, ${ }^{11}$ Unidad de Epidemiología Clínica, Hospital Infantil de México Federico Gómez-Facultad de Medicina Universidad Nacional Autónoma de México, Mexico City, Mexico, ${ }^{12}$ Departamento de Cirugía, Instituto Nacional de Ciencias Médicas y Nutrición Salvador Zubirán, Mexico City, Mexico, ${ }^{13}$ Center for Genomic Medicine and Diabetes Research Center (Diabetes Unit), Massachusetts General Hospital, Boston, Massachusetts, USA, ${ }^{14}$ Department of Medicine, Harvard Medical School, Boston, Massachusetts, USA, and ${ }^{15}$ Department of Radiology, I.M. Sechenov First Moscow State Medical

\begin{abstract}
Objective: A haplotype at chromosome 17p13 that reduces expression and function of the solute carrier transporter SLC16A11 is associated with increased risk for type 2 diabetes in Mexicans. We aim to investigate the detailed metabolic profile of SLC16A11 risk haplotype carriers to identify potential physiological mechanisms explaining the increased type 2 diabetes risk. Design: Cross-sectional study.

Methods: We evaluated carriers $(n=72)$ and non-carriers $(n=75)$ of the SLC16A11 risk haplotype, with or without type 2 diabetes. An independent sample of 1069 subjects was used to replicate biochemical findings. The evaluation included euglycemic-hyperinsulinemic clamp, frequently sampled intravenous glucose tolerance test (FSIVGTT), dual-energy X-ray absorptiometry (DXA), MRI and spectroscopy and subcutaneous abdominal adipose tissue biopsies. Results: Fat-free mass (FFM)-adjusted M value was lower in carriers of the SLC16A11 risk haplotype after adjusting for age and type 2 diabetes status $(\beta=-0.164, P=0.04)$. Subjects with type 2 diabetes and the risk haplotype demonstrated an increase of $8.76 \mathrm{U} / \mathrm{L}$ in alanine aminotransferase (ALT) $(P=0.02)$ and of $7.34 \mathrm{U} / \mathrm{L}$ in gammaglutamyltransferase (GGT) $(P=0.05)$ compared with non-carriers and after adjusting for gender, age and ancestry. Among women with the risk haplotype and normal BMI, the adipocyte size was higher $(P<0.001)$.
\end{abstract}

Published by Bioscientifica Ltd. 
Conclusions: Individuals carrying the SLC16A11 risk haplotype exhibited decreased insulin action. Higher serum ALT and GGT levels were found in carriers with type 2 diabetes, and larger adipocytes in subcutaneous fat in the size distribution in carrier women with normal weight.

\section{Introduction}

The Slim Initiative in Genomic Medicine for the Americas (SIGMA) Type 2 Diabetes Genetics Consortium identified a genome-wide significant association of a haplotype on chromosome $17 \mathrm{p} 13$ with increased risk of type 2 diabetes (1). This risk haplotype is highly prevalent in populations with a native American background (e.g. Mexican mestizos), in whom the risk variants are present in more than $25 \%$ of the population. The associated haplotype credible set includes four missense and one silent variant in SLC16A11 (V113I, L187L, D127G, G340S and P443T), as well as several non-coding variants in regulatory regions near the SLC16A11 gene. SLC16A11 is most highly expressed in thyroid, liver and salivary gland. The type 2 diabetes-associated variants lead to lower SLC16A11 expression in liver and also disrupt the interaction between SLC16A11 and basigin, a chaperone protein important for plasma-membrane localization of SLC16A11 (2). Together, these variant effects result in less SLC16A11 at the cell membrane, thus reducing SLC16A11 function. Knockdown of SLC16A11 expression in primary human hepatocytes alters fatty acid and lipid metabolism, leading to increases in intracellular acylcarnitine, diacylglycerol and triacylglycerol levels (2). These metabolic changes are also observed in experimental models of lipotoxicity and insulin resistance and as part of the pathophysiology of type 2 diabetes $(3,4)$.

The findings from these molecular and cellular studies implicate a role for SLC16A11 in hepatic metabolism and suggest primary effects on type 2 diabetes through insulin-resistant mechanisms; however, the physiologic mechanisms explaining the association of the SLC16A11 risk haplotype with hyperglycemia in humans have not been studied. Therefore, we conducted a deep phenotyping study in a sufficiently high number of risk haplotype carriers to comprehensively characterize the mechanisms through which variation in SLC16A11 contributes to the pathophysiology of type 2 diabetes. We hypothesized that carriers of the SLC16A11 risk haplotype will show lower insulin sensitivity in comparison with subjects without the risk haplotype.

\section{Subjects and methods}

We enrolled Mexican-mestizo men and women (with parents and grandparents born in Mexico), carriers (homozygous or heterozygous) or non-carriers of the risk haplotype at the SLC16A11, aged 20 to 79 years old, with a BMI between 18 and $34.9 \mathrm{~kg} / \mathrm{m}^{2}$. Individuals with type 2 diabetes with $\mathrm{HbA}_{1 \mathrm{c}}$ concentration $<8 \%$ and without insulin treatment were eligible for the study. No subject smoked tobacco, had cardiovascular disease, diabetes complications or an acute infection. Subjects with more than a 3\% weight loss in the last 3 months, taking medications or with conditions that could interfere with insulin secretion and action, high-performance athletes, with alcohol consumption more than 2 units per day in men or 1 unit in women were also excluded. Carriers and non-carriers were matched by gender, age ( \pm 5 years), BMI $\left( \pm 5 \mathrm{~kg} / \mathrm{m}^{2}\right)$ and in the type 2 diabetes group also by $\mathrm{HbA}_{1 \mathrm{c}}( \pm 1 \%)$. Subjects provided written informed consent before participating in this study, which was approved by the Comité de Ética en Investigación of the Instituto Nacional de Ciencias Médicas y Nutrición Salvador Zubirán (INCMNSZ). All procedures were in accordance with the ethical standards of the Helsinki declaration.

\section{Replication sample}

To confirm some of the associations between phenotypes and the SLC16A11 haplotype, we used a dataset that included an independent group of 1069 subjects in whom SLC16A11 genotype was known. This dataset is composed of subjects seeking attention at the Diabetes, Obesity, Internal Medicine or Dyslipidemia outpatient's Clinics at the INCMNSZ. The inclusion criteria were the same as in the discovery sample.

\section{Experimental procedures}

Studies were conducted at the Unidad de Investigación de Enfermedades Metabólicas (UIEM) at the INCMNSZ, 
whereas MRI studies were performed at the Centro Nacional de Investigación en Imagenología e Instrumentación Médica in Mexico City between 2015 and 2017. All evaluations were completed in the course of 1 month period.

To identify carriers of the risk haplotype of the SCL16A11 variant samples were genotyped using a Quant Studio 12K Flex Real-Time PCR platform from Thermo Fisher Scientific.

Body composition: Body fat mass (FM) and fat-free mass (FFM) were determined using dual-energy X-ray absorptiometry (DXA) (GE Healthcare). Subcutaneous and intra-abdominal adipose tissue volumes were quantified using MRI, and the subcutaneous/intraabdominal fat ratio was calculated. Intra-pancreatic and intrahepatic triglycerides content was determined using MRI spectroscopy (Philips Achieva 3 Teslas).

\section{Insulin sensitivity}

Participants were instructed to fast for $12 \mathrm{~h}$ before the study and admitted to the UIEM the day of the euglycemic-hyperinsulinemic clamp. Subjects with type 2 diabetes were instructed to suspend oral treatment 3 days before the procedure. The study was not performed if the fasting glucose concentration was $>250 \mathrm{mg} / \mathrm{dL}$. A catheter was inserted into a forearm vein to infuse dextrose and insulin, and a second catheter into a forearm vein in the contralateral hand was inserted in a retrograde fashion to obtain arterialized blood samples using a hot box. Insulin was infused at a rate of $50 \mathrm{mU} / \mathrm{m}^{2}$ body surface area (BSA)/min (initiated with a priming dose of $200 \mathrm{mU} /$ $\mathrm{m}^{2} / \mathrm{min}$ for $5 \mathrm{~min}$ and then $100 \mathrm{mU} / \mathrm{m}^{2} / \mathrm{min}$ for $5 \mathrm{~min}$ ). Euglycemia $(\sim 100 \mathrm{mg} / \mathrm{dL})$ was maintained by a variable infusion of $20 \%$ dextrose. During the clamp procedure, blood samples were drawn every $10 \mathrm{~min}$ during the final $30 \mathrm{~min}$ to determine glucose and insulin concentrations. Insulin sensitivity was determined as the glucose infusion rate ( $M$ value) during the final $30 \mathrm{~min}$ adjusted for weight and for the FFM (5).

Insulin secretory response: Participants were instructed to fast for $12 \mathrm{~h}$ before the frequently sampled intravenous glucose tolerance test (FSIVGTT). Two intravenous catheters were placed in antecubital veins (one in each arm). Blood samples were withdrawn at -10 , $-5,0,2,3,4,5,6,8,12,14,16,19,22,24$ and $25 \mathrm{~min}$ for measurement of serum glucose and insulin. Glucose was administered intravenously at a dose of $0.3 \mathrm{~g} / \mathrm{kg}$ for $60 \mathrm{~s}$ beginning at time 0 . The MINMOD Millenium computer package $(6,7)$ was used to estimate the acute insulin response to glucose (AIRg). The AIRg represents the acute insulin response and is defined as the area under the serum insulin curve between 0 and 10 min (7). The AIRg was adjusted by insulin sensitivity obtained in the clamp procedure ( $M$ value).

\section{Adipose tissue morphometric analysis}

Subcutaneous fat abdominal tissue biopsies were obtained nearby the umbilicus, in fasting conditions. For this report, adipocyte size was analyzed from subjects with normal weight. Thirteen controls (non-carriers: 7 women and 6 men) and 20 subjects with the SLC16A11 risk haplotype (carriers: 16 women and 4 men) were included in the analyses. Paraffin-embedded subcutaneous fat sections of $5 \mu \mathrm{m}$ thickness were mounted on poly- L-lysine pre-coated slides. After deparaffinization and rehydration, slides were stained with hematoxylin and eosin (SigmaAldrich). For each adipose tissue sample, 25 different fields were visualized with a Leica DM1000 LED (Leica Microsystems) microscopy and pictures taken in jpg format, using a LEICA ICC50 HD light microscope at 20x magnification. Subcutaneous fat cells were measured manually by delimiting the fat cell cross-sectional area in digital images using AxioVisio LE software real 4.8 versions (Zeiss copyright 2006-2010 Stuttgart-Germany). Data were obtained in 250 cells per subject. All histological measurements were performed by two independent observers without knowledge of the source of the tissues. Results are the averages of the two observers.

Laboratory methods: Plasma glucose concentration was measured by an automated glucose analyzer (Yellow Springs Instruments Co.). Serum insulin concentration was measured by using a chemiluminescent immunoassay (Beckman Coulter Access 2) and $\mathrm{HbA}_{1 \mathrm{c}}$ levels with HPLC (Variant II Turbo, Bio-Rad). Lipid concentrations (cholesterol, triglycerides and HDL cholesterol), apolipoprotein AI, apolipoprotein B, uric acid, creatinine, hepatic enzymes and $C$ reactive protein were measured using colorimetric assays (Unicel DxC 600 Synchron Clinical System Beckman Coulter). LDL cholesterol was calculated with the Friedewald equation when the triglyceride concentration was $<250 \mathrm{mg} / \mathrm{dL}$ (8). Thyroid-stimulating hormone (TSH) and free T4 were measured using electrochemiluminescence. For the TSH measurement a third-generation assay was used (Beckman Coulter). Plasma adiponectin, leptin and fibroblast growth factor (FGF)-21 concentrations were determined by performing ELISA assays (Merck Millipore). 


\section{Population stratification}

A principal components analysis was performed on the 32 ancestry informative markers genotypes, previously validated against whole genomic data using EIGENSTRAT software (9). The top two principal components were used as covariates in the linear regression model to correct for ancestry.

\section{Statistical analysis}

Sample size was estimated to have adequate power (between 80 and 90\%) to detect a difference between carriers and non-carriers of the SLC16A11 risk haplotype on the main variables: log-transformed $M$ value normalized for FFM and adjusted for the presence of type 2 diabetes; AIRg adjusted by age, sex and presence of type 2 diabetes and logtransformed ALT, AST and GGT. Continuous variables were tested for normality according the Kolmogorov-Smirnov test. Non-normally distributed variables are presented as medians and (interquartile ranges). Comparisons between carriers and non-carriers of the SLC16A11 haplotype were performed with Student's $t$ or Mann-Whitney $U$ tests, as appropriate. Bivariate correlations were evaluated using Spearman coefficients, and adjusted correlations were also performed. In order to assess the effect of genotype on insulin sensitivity (FFM-adjusted $M$ value) according diabetes status, stratified linear regression models adjusted for age were run. Before linear regression analysis nonnormally distributed variables were log-transformed. In the replication sample, the association between genotype and transaminases was assessed separately for individuals with and without diabetes through two-step regression models. In the first step, the outcome was regressed on age and gender. Residuals from this model were normalized using inverse normal transformation. In the second step, the residuals were taken as the outcome and regressed on genotype. Sensitivity of the model to outliers was assessed by comparing the coefficients obtained from the model with and without outliers. The assumptions of the linear regression model were checked via residual diagnostics. Analyses were performed using SPSS software, version 21 (SPSS Inc.) and RStudio version 3.3.2.

\section{Adipocyte size central tendency and data distribution}

Adipocyte size differences among groups: normal weight female non-carriers ( $n=1750$ cells/7 women) vs carriers ( $n=4000$ cells/16 women) and normal weight male noncarriers ( $n=1500$ cells/ 6 men) vs carriers $(n=1000$ cells $/ 4$ men) were compared by rank-sum test. Adipocyte size data from all groups were visualized in scatter plots.

\section{Results}

A total of 170 potential participants were screened for this study; 150 fulfilled the inclusion criteria and were invited to participate. Three participants withdrew consent and were discontinued. Seventy-five individuals were noncarriers and 72 were carries of whom 54 were heterozygous and 18 homozygous for the SLC16A11 risk haplotype.

\section{Metabolic characteristics}

Table 1 shows the general characteristics of the participants. The biochemical and anthropometric characteristics were similar in groups with and without the risk haplotype. No differences were observed in the lipid profile, $\mathrm{HbA}_{1 \mathrm{c}}$ levels, adipokines and markers of low-grade inflammation. The independent sample of 1069 (449 non-carriers and 620 carriers of the SLC16A11 risk haplotype) was composed of $25.2 \%$ individuals with type 2 diabetes with a median age of 44 years. Glucose and $\mathrm{HbA}_{1 \mathrm{c}}$ concentrations were higher in the carries of the SLC16A11 risk haplotype (Table 2).

\section{Insulin sensitivity}

Insulin action (evaluated by the clamp procedure) was significantly lower in carriers of the SLC16A11 risk haplotype: FFM-adjusted $M$ value 9.7 (7.5-13.2) vs 12.2 (9.4-15.3) $\mathrm{mg} / \mathrm{kg}$ FFM, $P=0.038$ (Fig. 1). Mean glucose and insulin concentrations at the end of the clamp were $99.5 \pm 2.8 \mathrm{mg} / \mathrm{dL}$ and $88.4 \quad(74.2-105.9) \mu \mathrm{UI} / \mathrm{mL}$ respectively. The risk SLC16A11 haplotype $(\beta=-0.164$, $P=0.048)$ and the presence of type 2 diabetes $(\beta=-0.333$, $P=0.003$ ) were significantly and independently associated with insulin sensitivity (FFM-adjusted $M$ value) in an additive model adjusted for age $\left(R^{2}=0.188, F=10.3\right.$, $P<0.0001)$. In the replication sample, we observed a higher HOMA2-IR adjusted for age, sex and FFM evaluated with bioelectrical impedance analyses (BIA) in carriers without type 2 diabetes compared to non-carrier individuals with borderline statistically significance $(\beta=0.2713, P=0.058)$.

\section{Liver enzymes}

Serum alanine transaminase (ALT) concentration was significantly higher in SLC16A11 risk haplotype 
Table 1 General characteristics of the carriers and noncarriers of the SLC16A11 risk haplotype.

\begin{tabular}{|c|c|c|c|}
\hline Variable & $\begin{array}{c}\text { Non-carriers } \\
(n=75)\end{array}$ & $\begin{array}{c}\text { Carriers } \\
(n=72)\end{array}$ & $\boldsymbol{P}$ \\
\hline Female sex & $38(55.1)$ & $40(54.8)$ & 0.957 \\
\hline Type 2 diabetes & $35(50.7)$ & 37 (50.7) & 0.996 \\
\hline Age, years & $45.5(28-57.8)$ & $43(29-56)$ & 0.977 \\
\hline Weight (kg) & $70.9 \pm 11.8$ & $72.2 \pm 12.8$ & 0.582 \\
\hline BMI $\left(\mathrm{kg} / \mathrm{m}^{2}\right)$ & $27.4 \pm 3.5$ & $28.1 \pm 4.0$ & 0.295 \\
\hline Glucose (mg/dL) & $96(87.5-114)$ & $98(88.5-110.5)$ & 0.670 \\
\hline $\mathrm{A} 1 \mathrm{C}(\%)$ & $5.7(5.3-6.3)$ & $5.6(5.3-6.2)$ & 0.698 \\
\hline Insulin $(\mu \mathrm{U} / \mathrm{mL})$ & $9.8 \pm 5.6$ & $9.2 \pm 6.0$ & 0.276 \\
\hline Triglyceride (mg/dL) & $122.5(82.5-202.0)$ & $122.0(94.0-159.0)$ & 0.488 \\
\hline Cholesterol (mg/dL) & $181.0 \pm 36.5$ & $177.3 \pm 35.4$ & 0.522 \\
\hline LDL-C (mg/dL) & $107.5 \pm 27.3$ & $104.9 \pm 30.6$ & 0.645 \\
\hline \multicolumn{4}{|l|}{ HDL-C (mg/dL) } \\
\hline Women & $45.5(40.0-51.5)$ & $46.5(39.3-55.5)$ & 0.779 \\
\hline Men & $36.0(30.0-44.0)$ & $39.0(34.5-46.0)$ & 0.231 \\
\hline Apo A (mg/dL) & $156.7 \pm 34.0$ & $151.4 \pm 31.0$ & 0.562 \\
\hline Apo B (mg/dL) & $96.3 \pm 25.3$ & $95.0 \pm 23.8$ & 0.725 \\
\hline Uric acid (mg/dL) & $5.6 \pm 1.3$ & $5.5 \pm 1.2$ & 0.983 \\
\hline Creatinine (mg/dL) & $0.77 \pm 0.25$ & $0.70 \pm 0.17$ & 0.141 \\
\hline ALT (U/L) & 20.5 (16.0-32.2) & $28.0(22.0-38.0)$ & 0.039 \\
\hline AST (U/L) & $23.0(18.0-29.0)$ & $26.0(22.0-34.0)$ & 0.067 \\
\hline GGT (U/L) & 16.5 (12.0-26.3) & $23.0(15.0-31.0)$ & 0.072 \\
\hline TSH (mIU/L) & $1.76(1.18-2.38)$ & $1.97(1.31-2.98)$ & 0.271 \\
\hline Free T4 (pmol/L) & $11.9(10.8-12.7)$ & $11.5(10.9-13.0)$ & 0.803 \\
\hline Adiponectin (ng/mL) & $7.6 \pm 3.7$ & $8.9 \pm 4.6$ & 0.102 \\
\hline Leptin (ng/mL) & $11.7(7.2-23.8)$ & $13.3(8.2-20.3)$ & 0.903 \\
\hline FGF-21 (ng/L) & $154.3(41.9-347.6)$ & $96.8(36.9-259.4)$ & 0.151 \\
\hline PCR (nmol/L) & $11.71(6.76-22.09)$ & $14.66(7.80-46.76)$ & 0.215 \\
\hline
\end{tabular}

carriers $(P=0.039)$. A non-significant similar trend was found for aspartate aminotransferase (AST) $(P=0.067)$ and gamma-glutamyl transferase (GGT) $(P=0.072)$. Subjects with diabetes and the $S L C 16 A 11$ risk haplotype demonstrated an increase of $8.76 \mathrm{U} / \mathrm{L}$ in ALT $(P=0.02)$ compared with non-carriers after adjusting for gender, age and ancestry. Similarly, carriers with type 2 diabetes had an increase of $7.34 \mathrm{U} / \mathrm{L}$ in GGT after adjusting for gender, age and ancestry $(P=0.05)$. The effect of the genotype was not significant in individuals without diabetes ( $P=0.448$ and $P=0.549$, respectively). No effect of the genotype was found for AST. To increase the power and to replicate the association, the hypothesis was tested in an independent sample. Carriers of the SLC16A11 risk haplotype with type 2 diabetes showed an increase in GGT and ALT in $11.2(P=0.03)$ and $7.9 \mathrm{U} / \mathrm{L}(P=0.01)$ respectively.

\section{Insulin secretory response}

In the analysis of the FSIVGTT, no difference in the AIRg was observed between carriers and non-carriers of the risk SLC16A11 haplotype in individuals with or without diabetes (263.2 (67.6-622.5) vs 260.9 (81.5-673.9),
Table 2 General characteristics of the carriers and non-carriers of the SLC16A11 risk haplotype in the replication sample.

\begin{tabular}{|c|c|c|c|}
\hline Variable & $\begin{array}{c}\text { Non-carriers } \\
(n=449)\end{array}$ & $\begin{array}{l}\text { Carriers } \\
(n=620)\end{array}$ & $\mathbf{P}$ \\
\hline Female sex & $298(60.0)$ & $392(63.6)$ & 0.252 \\
\hline Type 2 diabetes & $105(21.1)$ & $181(29.4)$ & 0.002 \\
\hline Age (years) & $44(29-55)$ & $44(32-54)$ & 0.606 \\
\hline Weight (kg) & $71.1(61.3-82)$ & 71.95 (61.8-81.9) & 0.913 \\
\hline BMI $\left(k g / m^{2}\right)$ & $27.4 \pm 4.8$ & $27.8 \pm 4.89$ & 0.169 \\
\hline Glucose (mg/dL) & $93(86-105)$ & 95 (87-109) & 0.030 \\
\hline $\mathrm{A} 1 \mathrm{c}(\%)$ & $5.5(5.2-6.0)$ & $5.6(5.3-6.2)$ & 0.004 \\
\hline Insulin $(\mu \mathrm{U} / \mathrm{mL})$ & 7.8 (4.9-11.3) & $7.90(5.30-12.5)$ & 0.263 \\
\hline Triglyceride (mg/dL) & $128(88.5-190.5)$ & 135 (93.0-200.0) & 0.110 \\
\hline Cholesterol (mg/dL) & $192.5 \pm 43.4$ & $188.4 \pm 41.4$ & 0.106 \\
\hline LDL-C (mg/dL) & $114(92.8-134)$ & 112 (89.4-134) & 0.224 \\
\hline \multicolumn{4}{|l|}{ HDL-C (mg/dL) } \\
\hline Women & $44.0(37.7-53.0)$ & $44.0(37.0-53.0)$ & 0.136 \\
\hline Men & $45.0(38.0-53.0)$ & $43.0(37.0-53.0)$ & 0.532 \\
\hline Apo B (mg/dL) & $103.8 \pm 27.7$ & $104.6 \pm 26.4$ & 0.665 \\
\hline Uric acid (mg/dL) & $5.2(4.3-6.1)$ & $5.2(4.4-6.3)$ & 0.658 \\
\hline Creatinine (mg/dL) & $0.72(0.61-0.86)$ & $0.71(0.59-0.84)$ & 0.197 \\
\hline $\operatorname{ALT}(\mathrm{U} / \mathrm{L})$ & $24.0(18-33)$ & $24.0(18.0-35.0)$ & 0.219 \\
\hline AST (U/L) & $23.0(20.0-28.0)$ & $23.0(20.0-28)$ & 0.807 \\
\hline GGT (U/L) & $20.0(14.0-29)$ & $20.0(14.0-32.0)$ & 0.247 \\
\hline
\end{tabular}

$P=0.910$ and $389.0(205.5-883.2)$ vs 541.5 (315.0-931.9) $\left.\mu \mathrm{U} / \mathrm{L}^{-1} \cdot \mathrm{min}^{-1}, P=0.331\right)$. Analyzing the disposition index (AIR multiplied by the $M$ value), no differences were found in individuals either with or without diabetes $(P=0.598$ and $P=0.162$, respectively).

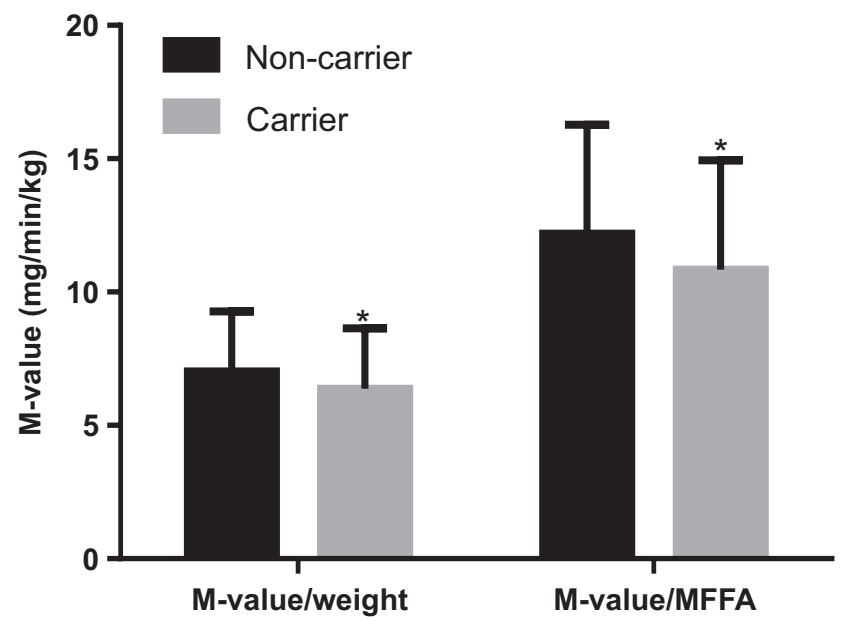

\section{Figure 1}

Comparison of median weight and fat-free mass adjusted $M$ values obtained by the euglycemic-hyperinsulinemic clamp between carriers $(n=75)$ and non-carriers $(n=72)$ of the risk SCL16A11 haplotype. Mann-Whitney $U$ test was run and a significantly lower $M$ value was observed in carriers of the risk haplotype for both weight and fat-free mass adjusted $M$ values $(P<0.05)$. 


\section{Adipose tissue histology}

To avoid the confounding effect of excess adiposity, analyses of adipose tissue histology were performed in participants with a BMI $\leq 25 \mathrm{~kg} / \mathrm{m}^{2}$. The distribution of the adipocyte size was shifted to higher values among risk haplotype carriers, an effect that was significant $(P<0.001)$ only in women. The proportion of total adipocytes represented by smaller-sized fat cells $(<5$ th percentile) was 10 times lower among the women with the risk haplotype, whereas the representation of big-sized fat cells (>95th percentile) was five times greater. Among men, only a two-fold increase in the proportion of the large-sized fat cells was found (Fig. 2).

\section{MRI spectroscopy assessment of intrahepatic, intra-pancreatic, intra-abdominal and subcutaneous fat}

No differences were found between carriers and noncarriers of the SCL16A11 risk haplotype in the intrahepatic, intra-pancreatic, intra-abdominal or subcutaneous fat content (Table 3). However, in the subgroup of women with BMI $\geq 30 \mathrm{~kg} / \mathrm{m}^{2}(n=17)$, carriers of the risk haplotype had a greater amount of intra-abdominal fat (147.3 vs $\left.64.0 \mathrm{~cm}^{2}, P=0.01\right)$ and a lower subcutaneous/intraabdominal fat ratio ( 4.9 vs $2.3, P=0.03$ ) than non-carriers. In the replication cohort, we observed significantly higher visceral fat content obtained from BIA in carriers compared to non-carriers adjusted for age, sex, type 2 diabetes status and FFM $(P=0.019)$.

\section{DXA assessment of body composition}

No difference in body composition was found between the risk haplotype carriers and non-carriers (Table 4). The mean or median FFM, FM and bone mineral content were not different between groups.

\section{Discussion}

The SIGMA Type 2 Diabetes Genetics Consortium has previously shown that a SLC16A11 variant haplotype confers $\sim 30 \%$ higher risk of type 2 diabetes per copy of the allele and is highly prevalent in populations
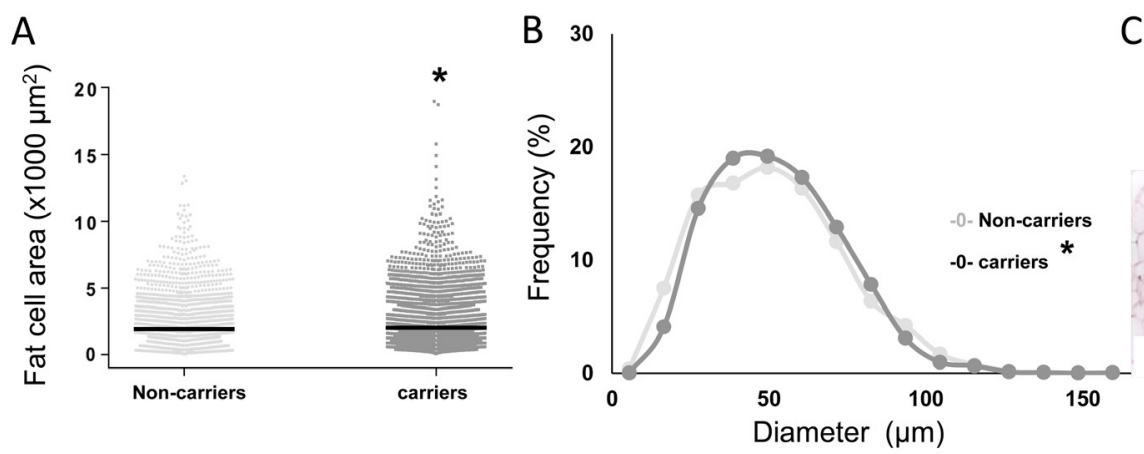

C

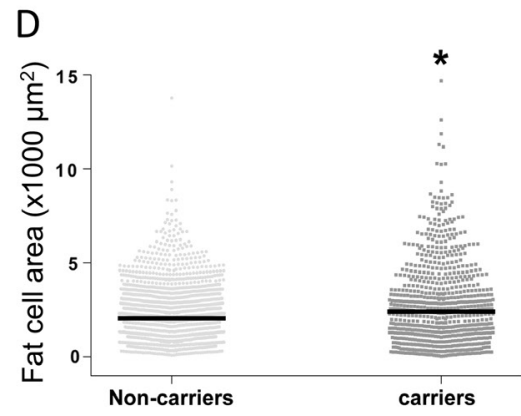

E
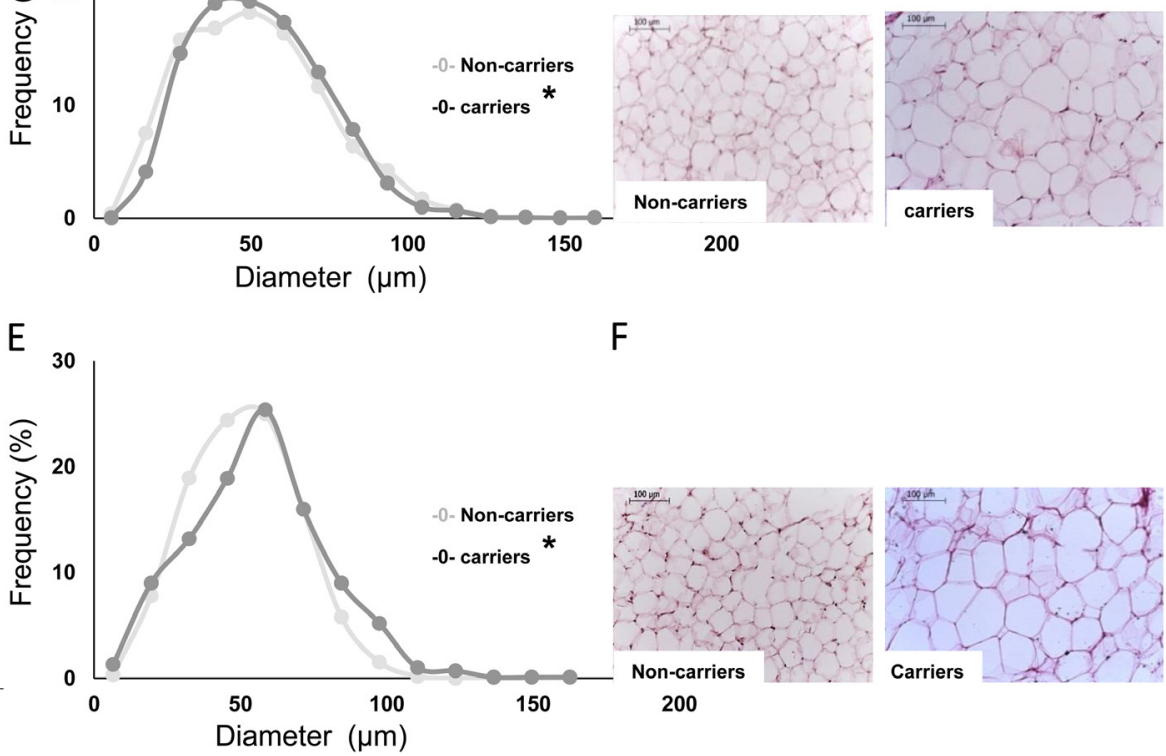

$\mathrm{F}$
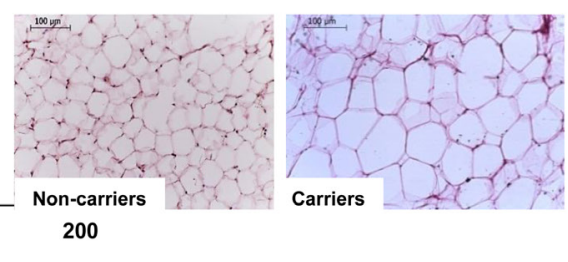

\section{Figure 2}

Adipose tissue histology between carriers and non-carriers of the SLC16A11 risk haplotype. A and D. Median adipocyte size and dispersion are represented in the scatter plots ((A) Female, (D) male)). (B and E) Relative frequency histograms for non-carriers (grey) and carriers (black) ((B) Female, (E) male)). (C and F) Microphotography of subcutaneous adipose tissue H\&E stained at 20x magnification ((C) Female, (F) male)) * $P<0.01$ carriers vs non-carriers. 
Table 3 Intrahepatic, intra-pancreatic, intra-abdominal and subcutaneous fat in carriers and non-carriers of the SLC16A11 risk haplotype.

\begin{tabular}{l}
\hline Variable \\
\hline Intrahepatic fat (\%) \\
Intra-pancreatic fat $(\%)$ \\
Intra-abdominal fat $\left(\mathrm{cm}^{2}\right)$ \\
Subcutaneous fat $\left(\mathrm{cm}^{2}\right)$ \\
Subcutaneous/intra-abdominal fat
\end{tabular}

\begin{tabular}{c}
\hline Non-carriers $(n=56)$ \\
\hline $2.0(0.68-5.8)$ \\
$2.4(1.1-8.2)$ \\
$78.4(51.0-109.7)$ \\
$205.0(175.0-305.0)$ \\
$2.9(1.9-4.9)$
\end{tabular}

\begin{tabular}{crcc}
\hline Carriers $(n=60)$ & & $\mathbf{P}$ \\
\hline $2.4(1.1-5.9)$ & & 0.346 \\
$2.9(1.3-6.7)$ & & 0.934 \\
$76.5(56.5-123.1)$ & & 0.493 \\
$237.8(184.2-315.2)$ & & 0.657 \\
$3.0(1.9-4.9)$ & 0.866 \\
\hline
\end{tabular}

with a native American background. Carriers of the risk haplotype develop type 2 diabetes at a younger age and with a lower BMI compared to non-carriers (1). In this report, we provide a detailed clinical profiling of SLC16A11 risk haplotype carriers. Individuals with the SCL16A11 risk haplotype have impaired insulin sensitivity and higher predominance of large diameter adipocytes in subcutaneous fat. The risk haplotype was associated with higher ALT and GTT in individuals with type 2 diabetes. These observations suggest that decreased SLC16A11 function changes hepatic and adipose tissue functionality, organs whose dysfunction condition type 2 diabetes pathophysiology.

SLC16A11 is a bidirectional solute carrier capable of transporting monocarboxylates such as pyruvate (10). While SLC16A11 transport of pyruvate has been experimentally shown, it may transport additional, as-yetunidentified substrates.

The haplotype under study is associated with decreased SLC16A11 function conferred by the combined effects of lower SLC16A11 expression in liver and reduced localization of SLC16A11 transporters at the cell membrane (2).

Increased hepatic de novo lipogenesis is a major source for the intra-hepatocellular lipids that cause lipotoxicity in type 2 diabetes (i.e. diacylglycerol and acylcarnitines), inducing insulin resistance $(11,12)$. The decreased insulin action found in risk haplotype carriers is consistent with findings from previous experimental studies, in which knockdown of SLC16A11 expression in primary human hepatocytes alters fatty acid and lipid metabolism resulting in an increase in intracellular acylcarnitines, diacylglycerols and triacylglycerol levels (2). Thus, it is expected that insulin resistance would be magnified in SLC16A11 risk haplotype carriers when they are exposed to a chronic caloric overload.

Cell transporters (mainly the related family member SLC16A11) export lactate from muscle or liver to other tissues (13). It can be hypothesized that lactate as a gluconeogenesis precursor may contribute to increased hepatic glucose production. Hence, this compensatory mechanism may contribute, in combination with the lipid-induced hepatic insulin resistance, to the appearance of hyperglycemia and type 2 diabetes. Furthermore, lactate is a lipolysis inhibitor by its interaction with GPR81. The receptor is activated in the presence of physiological lactate concentrations $(14,15)$. Chronic inhibition of lipolysis by lactate may contribute to the appearance of large-sized adipocytes, as found in the SLC16A11 risk allele carriers.

De novo lipogenesis in adipose tissue is reciprocally regulated with hepatic de novo lipogenesis. It is positively associated with insulin action and its activation protects against hepatic steatosis (16). De novo lipogenesis in adipocytes plays a role as an alternative source to store carbon molecules and calories besides the liver. Adipose tissue de novo lipogenic capacity is reduced in obese individuals, contributing to the metabolic abnormalities linked to excessive adiposity (17). The SLC16A11 deficiency may alter the balance between lipid synthesis in the fat and the liver. Additional studies are needed to confirm this hypothesis. Interestingly, we observed that women with the SLC16A11 risk haplotype have a greater amount of intra-abdominal fat and a lower subcutaneous/intra-abdominal fat ratio.

Table 4 Body composition and mineral content evaluated using DXA in carriers and non-carriers of the SLC16A11 risk haplotype.

\begin{tabular}{l} 
Variable \\
\hline Total mass $(\mathrm{kg})$ \\
Fat-free mass $(\mathrm{kg})$ \\
Fat mass $(\mathrm{kg})$ \\
Fat mass $(\%)$ \\
Bone mineral content $(\mathrm{g})$ \\
Visceral adipose tissue $\left(\mathrm{cm}^{3}\right)$ \\
Visceral adipose tissue $(\mathrm{g})$
\end{tabular}

\begin{tabular}{c} 
Non-carriers $(n=59)$ \\
\hline $70.5 \pm 11.5$ \\
$38.5(36.0-49.5)$ \\
$25.40 \pm 6.73$ \\
$37.25 \pm 7.74$ \\
$2271.0(2038.0-2653.0)$ \\
$1118.0(686.0-1667.0)$ \\
$1055.0(647.0-1572.0)$ \\
\hline
\end{tabular}

\begin{tabular}{cccc}
\hline Carriers $(n=64)$ & & $\boldsymbol{P}$ \\
$42.3 \pm 12.6$ & & 0.413 \\
$20.7(37.7-52.6)$ & & 0.346 \\
$26.15 \pm 7.31$ & & 0.553 \\
$37.40 \pm 7.63$ & & 0.916 \\
$2379.5(1923.0-2787.0)$ & & 0.671 \\
$1156.5(636.2-1821.7)$ & & 0.759 \\
$1090.5(600.2-1719.0)$ & 0.759 \\
\hline
\end{tabular}


Furthermore, among normal weight participants, the risk haplotype was associated with a remarkable increment in the number of large-sized adipocytes, an abnormality associated with adipose tissue dysfunction (18).

Another major finding of our study is the higher serum ALT concentrations found in the SLC16A11 risk haplotype carriers. Insulin resistance is a likely explanation for the abnormal ALT concentration, condition in which increased expression of $A L T 2$ has been reported $(19,20)$. An alternative explanation is hepatocellular damage due to hepatic steatosis. Though no difference in fat content was found between haplotype groups, the techniques used in humans in vivo may not be sensitive enough to capture intracellular lipid content. In addition, ALT catalyzes the transfer of an amino group from alanine to ketoglutarate in the cytoplasm, producing L-glutamate and pyruvate (21), potentially exacerbating haplotype-related cellular changes in pyruvate metabolism.

Limitations of the present study should be acknowledged including a relatively small sample size, although power was calculated for the main outcomes and this was sufficiently large and while we report nominal $P$ values, an independent sample was used to replicate the findings. Although statistically significant, the magnitude of the differences in the ALT concentrations and adjusted $M$ value between carriers and non-carriers of the risk haplotype was modest. However, greater differences are unlikely to occur in polygenic disorders (as type 2 diabetes). Therefore, the search for other contributors to the increased risk for type 2 diabetes in carriers of the SLC16A11 risk haplotype along with the confirmation of the findings in this work should continue. We did not perform clamps in the replication sample; therefore, we use the HOMA-IR index as a surrogate for the evaluation of insulin sensitivity. Nevertheless, we found a tendency for a higher insulin resistance in population with the risk haplotype without type 2 diabetes. We recognize that no single test is sufficient for a complete characterization of beta-cell function; however, the AIR represents the most widely used index. In addition, the AIR is dependent on insulin sensitivity thus comparison of AIR in populations with different insulin sensitivity may lead to inappropriate conclusions; therefore, we adjusted the AIR using the M value obtained in the clamp procedure. Finally, evaluation of the AIR allows an assessment of the first phase insulin secretion, and this is only one of the ways of response of the beta-cell and insufficient to characterize beta-cell function comprehensively. The relatively small number of homozygotes for the SLC16A11 risk haplotype precluded us to search for a dose-response relationship. Even though the histological analysis was performed in a small subset of the population, 250 cells from each subject were analyzed. In histological analysis, only normal weight subjects were included to avoid confusing changes due to overweight/obesity; therefore, these results might not be extrapolated. In addition, we did not evaluate selective hepatic insulin sensitivity, but we consider the high dose euglycemic-hyperinsulinemic clamp as the best approach for the overall evaluation of insulin resistance.

The detailed clinical characterization of the study sample and the successful matching process between carriers and non-carriers allowed us to detect subtle differences associated with the SLC16A11 risk haplotype. However, additional mechanistic studies and parallel exploration in relevant animal models are needed to explore the hypotheses here proposed for the phenotypes associated with SLC16A11 deficiency.

Individuals with the SLC16A11 risk haplotype have reduced insulin action. Subjects with type 2 diabetes and the risk haplotype demonstrated an increase serum ALT and GGT. In women carriers with normal weight the proportion of large-sized adipocytes in subcutaneous fat was higher. Additional studies are needed to describe the consequences of the SLC16A11 deficiency on the pathogenesis of the diabetes-related chronic complications and the response to therapy.

\section{Declaration of interest}

The authors declare that there is no conflict of interest that could be perceived as prejudicing the impartiality of this study.

\section{Funding}

The work was conducted as part of the Slim Initiative for Genomic Medicine, a project funded by the Carlos Slim Health Institute in Mexico and the Consejo Nacional de Ciencia y Tecnologia. Grant Infraestructura 255096. Alicia Huerta-Chagoya and Liliana Muñoz Hernández are funded through Cátedras CONACyT.

\section{Author contribution statement}

All authors contributed to experimental design, data acquisition and analysis and writing the manuscript. All authors approved the final version. P A wrote the manuscript and researched data. D V G researched data. O A C researched data. O Y B contributed to the manuscript. M D S contributed to the manuscript. $T \vee R$ researched data. A J M R researched data. C J B performed histological analyses. $\mathrm{L}$ M H researched data. I C B researched data. $\mathrm{H}$ M performed statistical analyses, reviewed/edited the manuscript. A H researched data. K G R researched data. G A W reviewed/ edited the manuscript. S B R J contributed to discussion and reviewed/ edited the manuscript. L E G P researched data. M L O researched data. $E R$ researched MRI data. J A researched MRI data. J F researched data. $\mathrm{P} C$ researched DXA data. $\mathrm{M} \mathrm{H} \mathrm{H}$ analyzed histological data. J C F contributed to discussion and reviewed/edited the manuscript. M T T L E Z researched V S contributed to discussion and reviewed/edited manuscript. C A A wrote the manuscript. CAS is the guarantor of this work. 


\section{Acknowledgements}

The authors thank Carmen Moreno, Adriana Cruz, Rosario RodríguezGuillén, Maribel Rodríguez-Torres, Saúl Cano-Colín and Guadalupe LópezCarrasco for technical assistance.

\section{References}

1 SIGMA Type 2 Diabetes Consortium, Williams AL, Jacobs SB, Moreno-Macias H, Huerta-Chagoya A, Churchhouse C, MarquezLuna C, Garcia-Ortiz H, Gomez-Vazquez MJ, Burtt NP et al. Sequence variants in SLC16A11 are a common risk factor for type 2 diabetes in Mexico. Nature 2014506 97-101. (https://doi.org/10.1038/ nature12828)

2 Rusu V, Hoch E, Mercader JM, Tenen DE, Gymrek M, Hartigan CR, DeRan M, von Grotthuss M, Fontanillas P, Spooner A et al. Type 2 diabetes variants disrupt function of SLC16A11 through two distinct mechanisms. Cell 2017170 199-212.e120. (https://doi. org/10.1016/j.cell.2017.06.011)

3 Muoio DM. Intramuscular triacylglycerol and insulin resistance: guilty as charged or wrongly accused? Biochimica et Biophysica Acta 20101801 281-288. (https://doi.org/10.1016/j. bbalip.2009.11.007)

4 Timmers S, Schrauwen P \& de Vogel J. Muscular diacylglycerol metabolism and insulin resistance. Physiology and Behavior 200894 242-251. (https://doi.org/10.1016/j.physbeh.2007.12.002)

5 DeFronzo RA, Tobin JD \& Andres R. Glucose clamp technique: a method for quantifying insulin secretion and resistance. American Journal of Physiology 1979237 E214-E223.

6 Bergman RN. Lilly lecture 1989. Toward physiological understanding of glucose tolerance. Minimal-model approach. Diabetes 198938 1512-1527. (https://doi.org/10.2337/diab.38.12.1512)

7 Bergman RN, Phillips LS \& Cobelli C. Physiologic evaluation of factors controlling glucose tolerance in man: measurement of insulin sensitivity and beta-cell glucose sensitivity from the response to intravenous glucose. Journal of Clinical Investigation $1981 \mathbf{6 8}$ 1456-1467. (https://doi.org/10.1172/JCI110398)

8 Friedewald WT, Levy RI \& Fredrickson DS. Estimation of the concentration of low-density lipoprotein cholesterol in plasma, without use of the preparative ultracentrifuge. Clinical Chemistry 197218 499-502.

9 Price AL, Patterson NJ, Plenge RM, Weinblatt ME, Shadick NA \& Reich D. Principal components analysis corrects for stratification in genome-wide association studies. Nature Genetics 200638 904-909. (https://doi.org/10.1038/ng1847)
10 Hediger MA, Clemencon B, Burrier RE \& Bruford EA. The ABCs of membrane transporters in health and disease (SLC series): introduction. Molecular Aspects of Medicine 201334 95-107. (https:// doi.org/10.1016/j.mam.2012.12.009)

11 Jones JG. Hepatic glucose and lipid metabolism. Diabetologia 201659 1098-1103. (https://doi.org/10.1007/s00125-016-3940-5)

12 Sanders FW \& Griffin JL. De novo lipogenesis in the liver in health and disease: more than just a shunting yard for glucose. Biological Reviews of the Cambridge Philosophical Society 201691 452-468. (https://doi.org/10.1111/brv.12178)

13 Brooks GA. Lactate shuttles in nature. Biochemical Society Transactions 200230 258-264. (https://doi.org/10.1042/bst0300258)

14 Brooks GA. Intra- and extra-cellular lactate shuttles. Medicine and Science in Sports and Exercise 200032 790-799. (https://doi. org/10.1097/00005768-200004000-00011)

15 Liu C, Wu J, Zhu J, Kuei C, Yu J, Shelton J, Sutton SW, Li X, Yun SJ, Mirzadegan T et al. Lactate inhibits lipolysis in fat cells through activation of an orphan G-protein-coupled receptor, GPR81. Journal of Biological Chemistry 2009284 2811-2822. (https://doi. org/10.1074/jbc.M806409200)

16 Roberts R, Hodson L, Dennis AL, Neville MJ, Humphreys SM, Harnden KE, Micklem KJ \& Frayn KN. Markers of de novo lipogenesis in adipose tissue: associations with small adipocytes and insulin sensitivity in humans. Diabetologia 200952 882-890. (https://doi. org/10.1007/s00125-009-1300-4)

17 Donnelly KL, Smith CI, Schwarzenberg SJ, Jessurun J, Boldt MD \& Parks EJ. Sources of fatty acids stored in liver and secreted via lipoproteins in patients with nonalcoholic fatty liver disease. Journal of Clinical Investigation 2005115 1343-1351. (https://doi. org/10.1172/JCI23621)

18 Smith U \& Kahn BB. Adipose tissue regulates insulin sensitivity: role of adipogenesis, de novo lipogenesis and novel lipids. Journal of Internal Medicine 2016280 465-475. (https://doi.org/10.1111/joim.12540)

19 de Luis DA, Aller R, Izaola O, Gonzalez Sagrado M, Conde R \& de la Fuente B. Role of insulin resistance and adipocytokines on serum alanine aminotransferase in obese patients with type 2 diabetes mellitus. European Review for Medical and Pharmacological Sciences 201317 2059-2064.

20 Maximos M, Bril F, Portillo Sanchez P, Lomonaco R, Orsak B, Biernacki D, Suman A, Weber M \& Cusi K. The role of liver fat and insulin resistance as determinants of plasma aminotransferase elevation in nonalcoholic fatty liver disease. Hepatology 201561 153-160. (https://doi.org/10.1002/hep.27395)

21 Vroon DH \& Israil Z. Chapter 99. Aminotransferases. In Clinical Methods: The History, Physical, and Laboratory Examinations. Ed WHHWH JW, 1990

Received 14 August 2018

Revised version received 20 October 2018

Accepted 19 November 2018 